\title{
A New Surgical Technique of Biliary Drainage
}

\author{
Shafiqul Hoque \\ Bangabandhu Sheikh Mujib Medical University, Dhaka, Bangladesh. \\ Email:shafiqul@dhaka.net
}

Received June $17^{\text {th }}$, 2013; revised July $16^{\text {th }}, 2013$; accepted August $2^{\text {nd }}, 2013$

Copyright (C) 2013 Shafiqul Hoque. This is an open access article distributed under the Creative Commons Attribution License, which permits unrestricted use, distribution, and reproduction in any medium, provided the original work is properly cited.

\begin{abstract}
Introduction: Roux-en-Y Hepatico-jejunostomy is the standard technique of biliary reconstruction after excision of choledochal cyst. Here the author describes a new surgical technique of biliary reconstruction using native gall bladder as biliary conduit. New Surgical Technique: The choledochal cyst is excised as standard technique but gall bladder with its neck is kept in situ. The gall bladder neck is anastomosed with the common hepatic duct stump and gall bladder fundus is anastomosed with the antero-inferior wall of the first part of distal duodenum. Materials \& Methods: Eleven patients with choledochal cyst have been operated with the new technique from July 2011 to December 2012 in the city of Dhaka, Bangladesh. Feeding was started from 3rd post-operative day and drain was removed by 7th day unless complicated and they were released from the hospital between 10 - 15 days. Results: The ages of eleven patients were from 3 months to 11 years. There were 7 females and 4 males. Lump was felt in 3 patients and jaundice was present in 4 patients. Recurrent abdominal pain was present in all patients. They were diagnosed by ultrasonography and MRCP was done in 6 patients only. Prothrombin time was elevated by 10\% \& 15\% in 2 patients. Average operation time was 2 hours and 10 minutes. A 9-year girl died suddenly and unexpectedly on the 5th post-operative day from severe convulsion of unknown origin. One child suffered from prolonged bile leakage and re-explored to repair anastomotic leak. Another patient had a collection near the anastomosis which resolved spontaneously. Discussion: Benefits of the new surgical technique are total absence of Roux-en-Y related intestinal complications. Possible disadvantages are discussed. It is anatomical and physiological. Small incision and less operation time are other benefits which need to be mentioned. Possible disadvantages are discussed.
\end{abstract}

Keywords: Biliary Reconstruction; Bilioenteric Anastomosis; Choledocho-Cholecystostomy; Cholecysto-Duodenostomy; Choledocho-Cholecysto-Duodenostomy

\section{Introduction}

Roux-en-Y hepatico-porto-jejunostomy (RYHJ) is now a well-accepted biliary drainage procedure for benign biliary tract diseases such as choledochal cyst and biliary strictures [1-5]. It involves two anastomoses, namely jejunojejunostomy and hepatico-porto-jejunostomy with a long segment $(40 \mathrm{~cm})$ of defunctioning jejunum as the biliary conduit. The anastomoses are quite big and normal anatomy is distorted. Here, the author describes an alternate procedure of biliary drainage which is anatomically as well as physiologically smaller in size and technically simpler. The technique has been successfully applied in 11 children with choledochal cyst.

\section{New Surgical Technique}

The main difference between this new technique and the original Roux-en-Y hepatico-porto-jejunostomy (RYHJ) is the preservation of gall bladder, which has been used as the biliary conduit. The abdomen was explored by smaller upper right transverse incision mostly dividing the rectus only. The area of dissection was only the sub hepatic region. The choledochal cyst was excised in the classical way (Figure 1). The important point in dissection is the isolation and preservation of the cystic artery. In one patient, the cystic artery was accidentally injured and ligated. But no adverse effect occurred since the gall bladder is well vascularized with alternate sources from the liver bed. The cystic duct was excised en-masse with the choledochal cyst at its junction with the neck of the gall bladder (Figure 2). The gall bladder fundus and part of body is mobilized from the liver bed such that the fundal tip easily comes close to the duodenum without any tension. The biliary channels in the liver were cleared of all stones, debris or sludge. Then the gall bladder neck was brought near the remaining common 


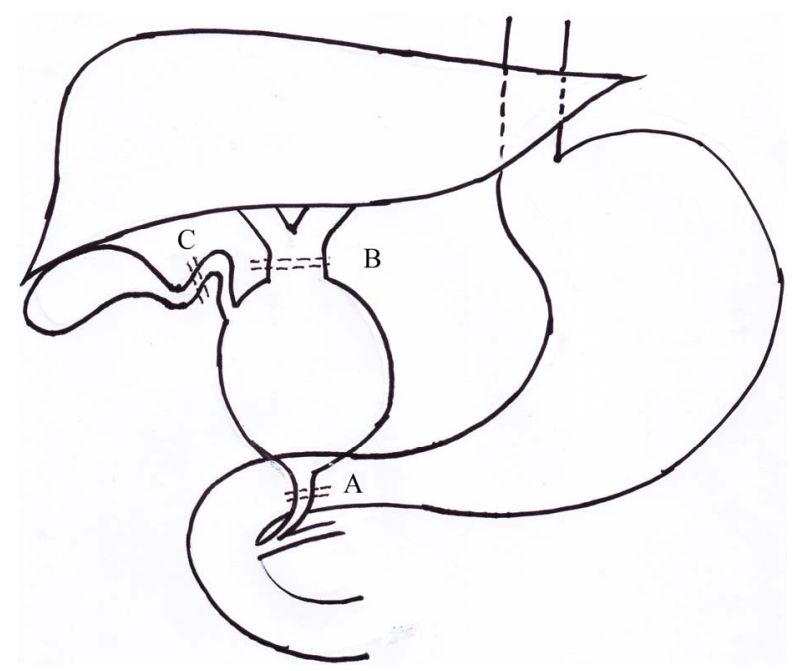

Figure 1. Choledochal cyst and lines of excision. A, division of lowest end of terminal common bile duct or choledochal cyst. B, Division at the site of common hepatic duct just above the choledochal cyst. $\mathrm{C}$, Division of gall bladder neck.

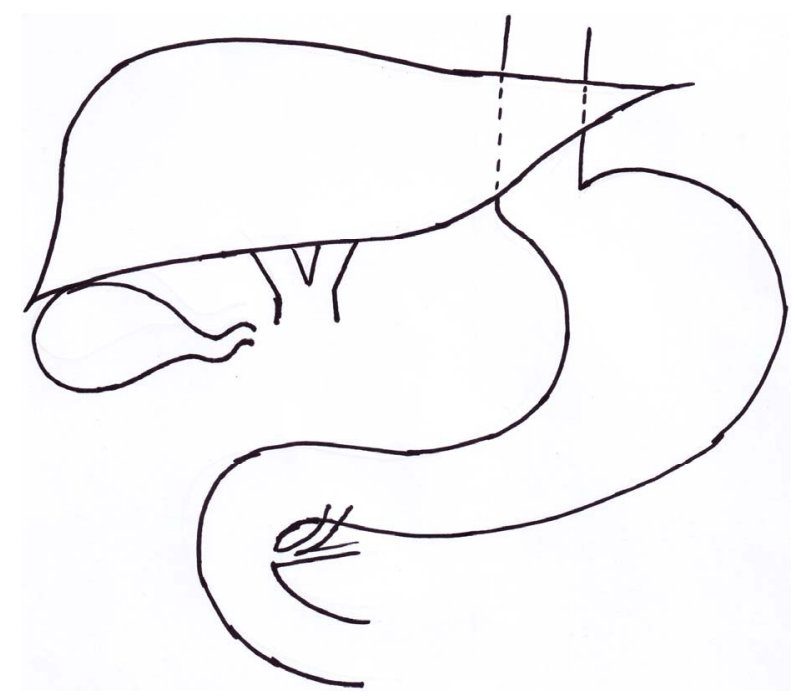

Figure 2. After the excision of the choledochal cyst.

hepatic duct for anastomosis. Any inequality was adjusted by opening the gall bladder neck longitudinally. Extra Care was taken not to twist the gall bladder neck. Interrupted water tight sutures with $6 / 0$ polyglycolic acid (Vicryl) were applied in a single layer. The site for the anastomosis of the fundal tip with duodenum was selected on the antero-inferior wall of distal first part of the duodenum. This site was specifically selected for possible prevention of reflux and subsequent cholangitis. The size of the anastomosis was kept within $10 \mathrm{~mm}$. The anastomosis was completed in two layers with interrupted stitches of 6/0 polyglycolic acid (Vicryl). The outer seromuscular stiches were taken little away from the margin such that the stoma invaginates a little into the duodenal lumen like a papilla to prevent possible reflux (Figure 3).

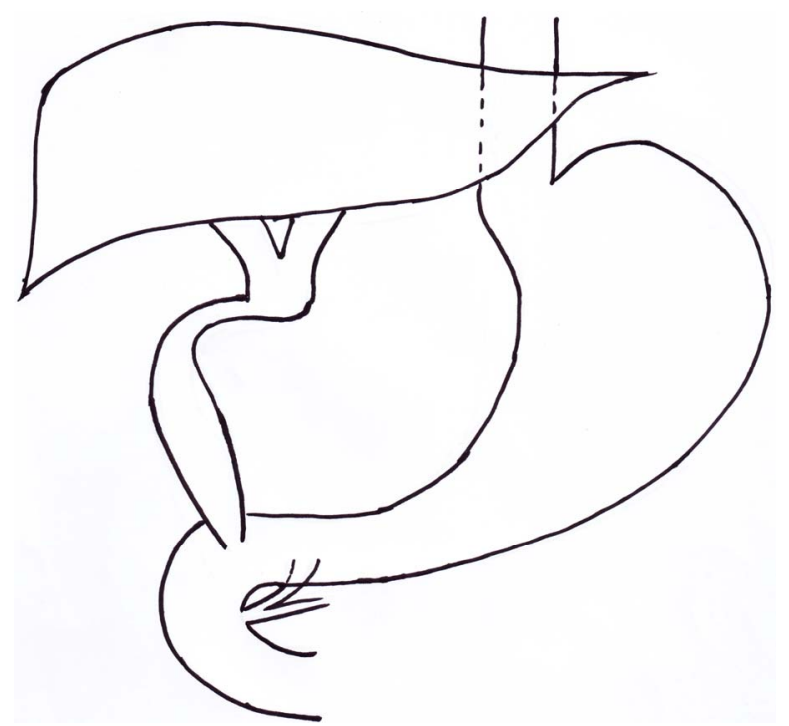

Figure 3. Anastomosis of gall blader neck with common hepatic duct and anastomosis of fundus of the gall bladder with antero-inferior wall of distal first part of duodenum.

The excised choledochal cyst was sent for histological examination. The cystic duct stump was also examined separately in 4 patients. Any leakage was checked. The wound was closed by giving a drain in the subhepatic pouch.

\section{Materials \& Methods}

This is a prospective study done in BSMMU (Bangabandhu Sheikh Mujib Medical University), DMCH (Dhaka Medical College Hospital) and private clinics in the City of Dhaka, Bangladesh during the period from July 2011 to December 2012. A total of 11 patients diagnosed as choledochal cyst were selected for the procedure. The procedure has been explained to the surgical team for a better assistance. Histological examination of the resected cyst specimen was done in all patients. Histology of the cystic duct stump was performed in 4 patients only. Oral feeding of liquids was given from the $3^{\text {rd }}$ post-operative day. The drain was kept usually for 7 days to observe for any leakage. If leakage is suspected the drainage tube was kept for prolonged period. Patients were discharged from the hospital between 10 - 15 days after the surgery. They were followed up at 6th \& 12th weeks after discharge from the hospital.

\section{Results}

A total of 11 patients with ages ranging from 3 months to 11 years were included in the study protocol. Seven patients were female and 4 were male. All the patients were diagnosed as choledochal cysts. Lumps were felt in 3 patients and jaundice was present in 4 patients. A variable degree of recurrent abdominal pain was present in all patients. All the patients were diagnosed by ultra- 
sonography. MRCP was done in 6 patients only. Early cirrhosis was found in only 3 patients. Small elevation of bilirubin level was seen in 4 patients. Prothrombin time was in- creased by $10 \%$ \& $15 \%$ in 2 patients. Blood transfusion was not needed in any of the patients. The average operation time was 2 hour \& 10 minutes. Uneventful recovery occurred in 8 patients. A 9-year-old girl was on smooth recovery until $5^{\text {th }}$ post-operative day midnight when she suffered severe convulsions and died within a short period. There were no abdominal signs and symptoms. The cause of death remains unexplained. One child suffered for prolonged biliary drainage and re-explored for anastomotic leakage at the site of porta hepatis and reconstructed again which resulted in an uneventful recovery. Another patient, a boy of 8 months old was recovering well until $7^{\text {th }}$ post-operative day when there was moderate abdominal distension with increase of drainage. Ultrasonography revealed a $3 \mathrm{~cm} \times 2 \mathrm{~cm}$ area of cystic swelling (collection) near the anastomotic site. The drain was kept in situ. Food intake was stopped and patient was kept under close observation. Food intake was restarted after 6 days and the patient responded well with resolution of the problem. Ultrasonography revealed absorption of the collection with no further external drainage. Thereafter the drainage tube was taken off. Malignancy was not found in any of the patient. Histology of the cystic duct stump showed abundance of fibres, scattered muscles with normal lining epithelium in 4 patients. The longest follow up is 15 months, with shortest follow up of 4 months in the last patient. All the 10 patients are doing well. None of the patients has suffered from attacks of abdominal pain with fever suggesting cholangitis nor any ulcer symptoms in this short period of follow up.

\section{Discussion}

Roux-en-Y hepatico-porto-jejunostomy (RYHJ) is a standard and well-practiced procedure for obstructive benign/ malignant biliary tract disease worldwide. It involves a bigger incision, longer operation time, two bigger anastomoses with greater possibility of intestinal complications. A long intestinal conduit for biliary drainage is another aspect. The complications of the original procedure are anastomotic stricture, recurrent calculi, recurrent cholangitis, malformed or twisted loop, too long or too short proximal jejunum and adhesions of intestinal loops [1-7].

The new technique uses native biliary channel for bile drainage instead of intestinal conduit. Gall bladder is not usually involved in the process of choledochal cyst formation. So gall bladder has been used as biliary conduit in place of Roux-en-Y Hepatico-jejunostomy. When the gall bladder was pulled down to anastomose with duodenum it more or less becomes an elongated tube with a little bigger caliber than normal CBD. It acts as a conduit for bile instead of a temporary reservoir of bile. But it is a biliary tract rather than intestinal tract as in Roux-en-Y hepatico-jejunostomy. In Roux-en-Y hepatico-jejunostomy the bile has to travel a long distance of $40 \mathrm{~cm}$ to come in contact with the food in the jejunum, whereas in present technique by traveling hardly a distance of $8-10 \mathrm{~cm}$, bile comes in contact with the food material in the duodenum like normal anatomy. Hence it is more physiological than Roux-en-Y anastomosis.

The advantages of the new technique are:

- The new technique maintains a normal anatomy more than the Roux-en-Y Hepatico-jejunostomy.

- A biliary conduit (gall bladder) has been used inplace of an intestinal conduit and hence more physiological.

- Area of dissection is small (only sub-hepatic region) thus reducing the area of adhesion.

- Primary incision is small.

- Possibility of reflux is less as the cholecysto-duodenostomy size is small $(<10 \mathrm{~mm})$ and its placement is on the anterior wall of the duodenum.

- Total operation time is less (130 min average) compared to Roux-en-Y hepaticojejunostomy (161 min to 5.1 hours) $[2,8]$.

- The possibility of gall stone formation is less as gall bladder works as a conduit with continuous flow of bile rather than a normal reservoir.

- The new technique logically excludes intestinal complications that may occur from Roux-en-Hepaticojejunostomy. The complications are adhesive obstruction, twisting of jejunal loop, duodenal ulcer [1-7].

No significant disadvantage or complications has been observed in the new technique comparing to all other bilio-enteric anastomoses. However possibility of occuring complications or disadvatages in the new technique are discussed.

- Anastomotic leakage: The anastomosis of dilated common hepatic duct and gall bladder neck is a weak one because of their thin walls specially GB neck. In the present technique it is suggested to give interrupted stiches with 3 - 5 mm gap by 6 - 0 polyglocolic acid (Vicryl) with round body needles. Importance of proper surgical technique in reducing complications in biliary reconstruction has been stressed by Satoshi et al. [9]. Gentle handling is very important. In the present series 2 patients presented with complication of bile leakage. Re-exploration and repair of the leak has been done successfully in one patient and leakage spontaneously stopped in another. Anastomotic leakage is a documented complication in bilio-enteric/ biliary duct-to-duct anastomosis [3,7,10-12].

- Anastomotic stricture: It is another common complication of biliary tract surgery [3,10,13-17]. In the present technique dilated hepatic duct is anastomosed 
with narrow gall bladder neck where it may be complicated by stricture. Even it happened it can be treated by a redo-cholecystostomy with wider part of gall bladder body or Roux-en-Y choledocho-jejunostomy.

- Biliary fistula: An anastomotic leakage if not closed spontaneously or not repaired in time it will result in a biliary fistula. It is not a common complication. Biliary fistula as a complication is found in one report of Roux-en-Y Hepaticojejunostomy [3].

- Reflux and Recurrent cholangitis: It is a common complication in bilio-enteric/biliary duct-to-duct anastomosis [3,5,10,13,14,17]. When the normal anatomy of protruding papilla and sphincter of Oddi at termination of CBD (Common Bile Duct) is replaced by simple anastomosis there is possibility of reflux of foods to cause cholangitis. A long isoperistaltic segment of jejunum $(>40 \mathrm{~cm})$ in Roux-en-Y anastomosis or an interposition of reconstructed valve is a good prevention for reflux. Even then there is reflux to cause cholangitis $[2,3,5,13,14,17]$. But in choledochoduodenostomy no such mechanism works and hence incidence of reflux is more [14]. This high incidence of reflux is probably due to the fact that the short common hepatic duct is anastomosed directly with the superior surface of duodenum which has been pulled up making an angulation and easy way for reflux. Also to add that the anastomosis with duodenum is wide because of dilated common hepatic duct from obstructive effect of the choledochal cyst. In the present technique a small anastomosis with some degree of projecting stoma into the anterior wall of first part of duodenum is likely to minimise the incidence of reflux.

- Gall stones: Gall stone is an uncommon complication $[6,13]$ which may occur in the present technique as because native gall bladder is preserved. But in the new technique, the possibility is less as the gall bladder has been used as a conduit where there is continous flow of bile instead of a normal reservoir.

- Carcinoma of gall bladder: If cyst wall with the lining epithelium is retained (as in earlier days) either in partial excision or cystoduodenostomy there is good chance of carcinoma formation from the abnormal cyst epithelium. But in presnt technique the cyst has been completely removed. So chance of developing carcinoma from cyst remnant does not arise. But, as gall bladder is retained the possibility of developing carcinoma from it is always there like a normal gall bladder.

\section{Conclusion}

The new surgical technique is more anatomical and physiological than standard Roux-en-Y hepatico-jejunostomy. There was no significant disadvantage or com- plication within this short period of follow-up. However, a long term follow-up of patients is needed to look for future probable complications of stricture, recurrent cholangitis or any other untoward complications. Even such complications occur there is scope for a redo-surgery or a Roux-en-Y Hepaticojejunostomy. The technique can be employed not only for the treatment of choledochal cyst but also for the treatment of other causes of Common Bile Duct replacement.

\section{Acknowledgements}

I would like to acknowledge my wife, Dr. Nasreen Raushan, for her contributions in editing and proofreading the paper and for her constant support.

\section{REFERENCES}

[1] Z. Li, N. Cui and L. Chen, "Treatment Experience of Subsequent Complications after Roux-en-Y Biliojejunostomy,” European Surgical Research, Vol. 43, No. 1, 2009, pp. 34-38. doi:10.1159/000216521

[2] E. Elhalaby, A. Hashish, M. Elbarbary and M. Elwagih, "Roux-en-Y Hepaticojejunostomy versus Hepaticoduodenostomy for Biliary Reconstruction after Excision of Choledochal Cysts in Children,” Annals of Pediatric Surgery, Vol. 1, No. 1, 2005, pp. 79-85

[3] P. Stefanini, M. Carboni, N. Patrassi, A. Basoli, G. de Bernardinis and P. Negro, "Roux-en-Y Hepaticojejunostomy: A Reappraisal of Its Indications and Results," Annals of Surgery, Vol. 181, No. 2, 1975, pp. 213-219. doi:10.1097/00000658-197502000-00016

[4] G. Pappalardo, S. Correnti, S. Mobarhan, P. Trentino, A. Pietropaolo, F. Frattaroli and G. Castrini, "Long-Term Results of Roux-en-Y Hepaticojejunostomy and Hepaticojejunoduodenostomy," Annals of Surgery, Vol. 196, No. 2, 1982, pp. 149-152. doi:10.1097/00000658-198208000-00006

[5] A. Shimotakahara, A. Yamataka, T. Yanai, H. Kobayashi, T. Okazaki, G. J. Lane and T. Miyano, "Roux-en-Y Hepa tico-Jejunostomy for Biliary Reconstruction during the Surgical Treatment of Choledochal Cyst: Which Is Better?” Pediatric Surgery International, Vol. 21, No. 1, 2005, pp. 5-7. doi:10.1007/s00383-004-1252-1

[6] G. Halff, S. Todo, R. Hall and T. E. Starzl, "Late Complications with Gall Bladder Conduit Biliary Reconstruction after Liver Transplantation,” Transplantation, Vol. 48, No. 3, 1989, pp. 537-539.

doi:10.1097/00007890-198909000-00044

[7] K. Congo, M. F. Lopes, P. H. Oliveira, H. Matos, S. Basso and A. Reis, "Outcomes of Choledochal Cysts with or without Intrahepatic Involvement in Children after Extrahepatic Cyst Excision and Roux-en-Y Hepaticojejunostomy," Annals of Hepatology, Vol. 11, No. 4, 2012, pp. 536-543.

[8] M. T. Santore, B. J. Behar, T. A. Blinman, E. J. Doolin, H. L. Hedrick, P. Mattei, M. L. Nance, N. S. Adzick and A. W. Flake, "Hepaticoduodenostmy versus Hepaticojeju- 
nostomy for Reconstruction after Resection of Choledochal Cyst,” Journal of Pediatric Surgery, Vol. 46, No. 1, 2011, pp. 209-213. doi:10.1016/j.jpedsurg.2010.09.092

[9] S. Hirano, E. Tanaka, T. Tsuchikawa, J. Matsumoto, T. Shichinohe and K. Kato, "Technique of Biliary Reconstruction Following Bile Duct Resection,” Journal of Hepatobiliary-Pancreatic Sciences, Vol. 19, No. 3, 2012, pp. 203-209. doi:10.1007/s00534-011-0475-5

[10] N. Akamatsu, Y. Sugawara and D. Hashimoto, "Biliary Reconstruction, Its Complications and Management of Biliary Complications after Adult Liver Transplantation: A Systematic Review of the Incidence, Risk Factors and Outcome,” Transplant International, Vol. 24, No. 4, 2011, pp. 379-392. doi:10.1111/j.1432-2277.2010.01202.x

[11] F. Cianci, F. Al Oraifi, F. Tareen and M. Corbally, "Choledochal Cyst: Our Ten Year Experience,” Irish Medical Journal, Vol. 105, No. 4, 2012, pp. 110-113.

[12] S. S. Saluja, M. Nayeem, B. C. Sharma, G. Bora and P. K. Mishra, "Management of Choledochal Cysts and Their Complications,” American Surgeons, Vol. 78, No. 3, 2012, pp. 284-290.

[13] A. J. Oishi, M. G. Sarr, D. M. Nagomey, M. D. Traynor and P. Mucha Jr., "Long-Term Outcome of Cholecysto- enterostomy as a Definitive Biliary Drainage Procedure for Benign Disease,” World Journal of Surgery, Vol. 19, No. 4, 1995, pp. 616-619. doi:10.1007/BF00294736

[14] D. P Vogt and R. E Hermann, "Choledochoduodenostomy, Choledochojejunostomy or Sphincteroplasty for Biliary and Pancreatic Disease," Annals of Surgery, Vol. 190, No. 2, 1981, pp. 161-168.

[15] R. M. Walsh, J. M. Henderson, D. P. Vogt and N. Brown, "Long-Term Outcome of Biliary Reconstruction for Bile Duct Injuries from Laparoscopic Cholecystectomies," Surgery, Vol. 142, No. 4, 2007, pp. 450-456. doi:10.1016/j.surg.2007.07.008

[16] R. F. Saidi, N. Ilias, D. Sc Ko, T. Kawai, J. Markmann, A. B. Cosimi and M. Hartl, "Biliary Reconstruction and Complications after Living-Donor Liver Transplantation,” HPB (Oxford), Vol. 11, No. 6, 2009, pp. 505-509. doi:10.1111/j.1477-2574.2009.00093.X

[17] B. Mukhopadhyay, R. M. Shukla, M. Mukhopadhyay, K. C. Mandal, P. P. Mukharjee, D. Roy, S. K. Biswas and K. S. Basu, "Choledochal Cyst: A Review of 79 Cases and the Role of Hepaticodochoduodenostomy," Journal of Indian Association of Pediatric Surgeons, Vol. 16, No. 2, 2011, pp. 54-57. doi:10.4103/0971-9261.78131 